\title{
Labyrinthe
}

29 | 2008 (1)

Ce que le libéralisme promet

\section{Résister à la « crise de la conscience historique »}

Entretien

\section{Sophie Wahnich}

\section{CpenEdition}

Journals

Édition électronique

URL : http://journals.openedition.org/labyrinthe/3483

DOI : $10.4000 /$ labyrinthe.3483

ISSN : 1950-6031

Éditeur

Hermann

Édition imprimée

Date de publication : 11 janvier 2008

Pagination : 105-120

ISBN : 9782952613163

Référence électronique

Sophie Wahnich, «Résister à la « crise de la conscience historique » », Labyrinthe [En ligne], 29 | 2008

(1), mis en ligne le 11 janvier 2010, consulté le 17 novembre 2020. URL : http://

journals.openedition.org/labyrinthe/3483 ; DOI : https://doi.org/10.4000/labyrinthe.3483

Propriété intellectuelle 


\title{
RÉSISTER À LA «CRISE DE LA CONSCIENCE HISTORIQUE»
}

\author{
Entretien \\ avec Sophie Wahnich \\ Réalisé, le 19 avril 2007, par Déborah Cohen et David Schreiber
}

\begin{abstract}
Historienne, Sophie Wahnich est chargée de recherche au Centre national de la recherche scientifique. Labyrinthe a souhaité la rencontrer car sa pratique déplace les cadres ordinaires de son métier. Jeu temporel tout d'abord : spécialiste de la Révolution française, elle ne s'interdit jamais de confronter son savoir à des enjeux contemporains, qu'il s'agisse des guerres du début du $\mathrm{Xx}^{\mathrm{e}}$ siècle, de celles de l'ex-Yougoslavie ou des formes de revendications les plus récentes. Jeu ensuite avec le matériau d'où naît le sens : partie d'une analyse de discours, Sophie Wahnich affronte désormais des objets aussi divers que les organisations muséales, les gestes et les rituels. Jeu enfin avec les disciplines, où l'histoire se fait notamment voisine de l'anthropologie et de la philosophie. Cette labilité des objets analysés et des instruments de recherche, Sophie Wahnich la met essentiellement au service d'une compréhension fine des formes historiques de la souveraineté et des manières d'élaborer le désaccord selon différents régimes émotifs. Son prochain livre, La Longue Patience du peuple. 1792, naissance de la République, est à paraître chez Payot, en février 2008.
\end{abstract}

LABYRINTHE - Comment êtes-vous entrée en histoire? Et pourquoi avoir choisi l'histoire révolutionnaire?

Sophie Wahnich - Pourquoi l'histoire? Je pense que ce n'était pas un choix libre, au sens où un sujet libre déciderait de son métier. Sans doute ce «faire de l'histoire», cette condition d'historien étaientils requis au sein de la famille, noués à l'histoire familiale, même si 
cette requête relevait d'une sorte de déni; un déni qui affleure et auquel il faudrait donner un nom et une forme. Mes parents ont refusé que ma sœur fasse de l'histoire, parce que, disaient-ils, ce n'était pas une discipline qui permettait d'avoir un métier. Ils l'ont convaincue de faire du droit. Mon frère a tourné autour de l'histoire en questionnant d'une manière frontale le fait politique, mais je l'ai souvent entendu parler des Républicains espagnols, de Vichy, du temps présent comme d'effets de cette histoire tragique du $\mathrm{xx}^{\mathrm{e}}$ siècle. Et puis moi, troisième dans la fratrie, je me suis inscrite en histoire en prétextant des équivalences de classe préparatoire; ça a fait un drame, mais ma mère a fini par céder. Au bout du compte, le refus de ma mère était noué, à mon avis, à ce qui nous motivait tous : essayer de comprendre des choses très lourdes - la Seconde Guerre mondiale, l'extermination des juifs par les nazis, pourquoi la violence, l'humanité et l'inhumanité.

Et pourquoi l'histoire de la Révolution? Sans doute parce qu'il y a quelque chose de très vif dans l'histoire de la Révolution, et que c'est justement un antidote. D'abord très concrètement, par la matérialité de l'archive : il y a un mouvement de la vie politique et de la vie tout court qui passe dans la matière même de l'archive et de ce qui se fait dans la Révolution. Et puis aussi antidote évidemment parce que la Révolution comprenait l'espoir de transformation, la possibilité d'inventer des formes sociales qui ne seraient pas celles, justement, de la Seconde Guerre mondiale en France ou du nazisme.

Quand j'ai choisi, je ne savais pas d'où venait ce choix. Aujourd'hui, je suis absolument sûre que telles sont les raisons.

LABYRINTHE - Au moment où vous commencez à faire de la recherche, c'est la fin des années 1980. Votre maîtrise date de 1986, c'est un moment où la Révolution française est au cœur de nombreux débats extra-universitaires.

SoPHIE WAHNich - Oui, mais à ce moment-là je n'en ai pas non plus une claire conscience. Je sais que je veux travailler à la Sorbonne et pas du côté de François Furet, mais si je choisis Paris I, c'est en tant qu'université réputée de gauche. Je continue sur une lancée qui me semble évidente. D'ailleurs, j'ai hésité entre un travail sur le XVIII ${ }^{\mathrm{e}}$ ou sur le $\mathrm{XIX}^{\mathrm{e}}$ siècle. Je voulais travailler sur les cercles Proudhon pour tenir, sans le savoir, les deux bouts, c'est-à-dire comprendre comment ceux qui prétendaient à la révolution et à l'émancipation avaient produit des formes de fascisme. J'étais allée voir Maurice Agulhon, 
qui partait au Collège de France et m'avait orientée vers son collègue Michel Vovelle. J'avais déjà envisagé un sujet possible en histoire de la Révolution parce que j'avais été frappée par un texte de Saint-Just sur les factions de l'étranger, du 23 ventôse an II, et par tout ce qu'on pouvait saisir de cette notion d'étranger dans la période révolutionnaire. J'ai finalement fait une maitrise en histoire de la Révolution dans ce que je pensais être le bon camp idéologique.

J'avais auparavant, en classes prépas, hésité entre littérature et histoire, et évidemment j'avais des regrets. Mais il y avait autour de l'histoire quelque chose de conditionné qui faisait qu'il fallait le faire. Je me lançai donc pour ma maîtrise dans l'analyse de discours : j'avais le sentiment que je réconciliais ainsi le rapport à la langue et l'histoire.

\section{LABYRINTHE - Ce n'était pourtant pas tellement l'optique de Michel Vovelle.}

SophiE WAHNich - En effet, ce fut Jacques Guilhaumou qui suivit de près mon travail. Mais un aspect intéressait Michel Vovelle : l'Institut d'histoire de la Révolution française publiait (et publie toujours) les Archives Parlementaires, qui constituèrent mon corpus. D'autre part, en maîtrise, j'ai vraiment joué le jeu de Michel Vovelle, c'est-àdire que j'ai fait de l'histoire quantitative cartographiée. Donc je donnais satisfaction à la fois à mes différents interlocuteurs et à mon propre désir. Et j'étais absolument dans le ravissement naïf de croire que parce que je travaillais sur la notion d'étranger et non pas sur les juifs à l'époque de la Révolution française, j'avais échappé à toute détermination. J'avais tout juste vingt ans, j'étais assez naïve, mais en même temps avec la force que donne la naïveté.

LABYRINTHE - Mais ce moment-là est aussi celui de la pleine époque d'un rapport de force au sein de cette historiographie révolutionnaire, avec Paris I d'un côté et le révisionnisme de l'autre 1 .

SophiE WaHNich - Moi je défendais Soboul et ses héritiers à l'époque - je le défends toujours d'ailleurs, je pense qu'il est l'auteur d'une

1. On se souvient que les années 1980 ont vu la montée en puissance d'une historiographie dite «révisionniste» qui, autour de François Furet et de Mona Ozouf en particulier, accorda une nouvelle importance à la culture politique et, inscrivant la Révolution dans un ensemble de mouvements responsables de la naissance du totalitarisme, récusa les interprétations qui, dans la lignée de Soboul, s'inspiraient du marxisme. Voir la récente traduction en français des articles de Eric J. Hobsbawm sur le sujet : Aux armes, historiens. Deux siècles d'histoire de la Révolution française, Paris, La Découverte, 2007, trad. Julien Louvrier. 
grande œuvre. J'étais au bon endroit pour le faire. Je défendais l'Institut d'histoire de la Révolution française, où je passais mes journées de travail, où je racontais mes échecs au permis de conduire et ma réussite au CAPES à Michel Vovelle qui trouvait ça très drôle. Les historiens qui gravitaient autour de François Furet semblaient fort loin. Il y avait peu de gens du côté de la Sorbonne qui souhaitaient des rencontres. Mais c'est surtout Furet qui ne venait pas aux réunions de préparation du Bicentenaire. Il était systématiquement invité, pourtant. Il y a également eu des tentatives pour organiser des débats publics... Je me souviens en particulier que Jacques Guilhaumou avait voulu en organiser un : François Furet s'était dédit au dernier moment et ça n'a pas eu lieu. Le camp Furet n'avait aucun intérêt à accepter le débat puisqu'il tenait la presse, avec le Nouvel Obs notamment. La Sorbonne avait la responsabilité de la préparation du Bicentenaire, mais eux se donnaient l'image d'être en marge des représentations officielles, ce qui les maintenait dans une représentation de modernité, par rapport à la Sorbonne ringarde. Donc, n'ayant aucun intérêt à frayer avec nous, ils ne frayaient pas.

$\mathrm{Au}$ moment des colloques, il y eut cependant quelques échanges. Je me souviens, sur la question de la mémoire et de l'héritage, d'un échange contradictoire entre Patrick Garcia et Mona Ozouf. Je crois que c'est à ce moment-là que Mona Ozouf a dit qu'on pouvait faire son marché dans la Révolution, choisir ce qu'on voulait, laisser le reste. On ne parlait pas encore de droit d'inventaire, mais c'était un peu le même genre de chose.

LABYRINTHE - Comment, au-delà de vos premières recherches, et notamment de votre thèse, s'est poursuivie la réflexion sur le lien passéprésent, qui apparaît comme un fil rouge dans votre recherche?

SophiE WAHNich - Après la thèse, on sort de l'innocence et de la naïveté. Je n'était plus fascinée par la science positive et quantitative, et donc, à l'issue du trajet de formation par la thèse, j' avais même le sentiment ou peut-être la conviction que ce n'était pas par des méthodes scientifiques au sens où on pouvait les entendre dans l'analyse de discours ou même dans les sciences sociales aujourd'hui qu'il y avait une possibilité de poser les questions qui m'intéressaient et de transmettre quelque chose. Je pensais que la construction des espaces scientifiques se faisait de manière très close, que ça pouvait être fascinant, enthousiasmant du point de vue du jeu, comme jeu mathémati- 
que, mais que en tant que tel, le savoir scientifique pouvait être vain, ne rien transmettre, n'avoir aucun effet dans le monde, sur le monde. J'avais un sentiment très ambivalent à l'égard de l'histoire comme science et j'étais ravie de lire Jacques Rancière et ses Mots de l'histoire $^{2}$ qui redonnaient toute leur place aux tours de littérature dans la production de l'histoire. Tout en proposant une conception de la science comme lieu où la quête est celle du vrai, il autorisait l'articulation d'une dimension littéraire et politique au sein d'un savoir qui pour autant revendiquerait tout de même le nom de science. Il ne s'agissait pas de renoncer au contrat scientifique mais de le subvertir ou de le ranimer par ces déplacements.

Cependant, le moment fondateur le plus lumineux fut pour moi l'article de Nicole Loraux sur l'éloge de l'anachronisme'. C'est un article de 1993 et moi je soutenai ma thèse en $1994^{4}$. Au moment où je l'ai lu, cet article est venu étayer une démarche que je croyais solitaire. J'étais partie d'une phrase de Saint-Just, «il faut que vous fassiez une cité, c'est-à-dire un peuple qui soit ami hospitalier et frère », pour questionner la notion d'étranger en Révolution. J'avais construit des couples amitié/trahison, hospitalité/suspicion, fraternité/exclusion qui étaient au cœur de l'actualité. Je n'avais pas construit ma thèse dans une logique d'érudition, j'avais utilisé l'érudition en histoire de la Révolution pour comprendre quelque chose sur le présent. Je savais que c'était les enjeux passé-présent qui m'intéressaient, mais j'avais une hésitation sur les manières de le dire et de ne pas le dire. Après avoir lu l'article de Nicole Loraux, je me suis sentie plus libre d'essayer de faire de l'histoire comme science du présent, à la manière de Walter Benjamin. Je me suis autorisée à construire un plan particulier et surtout à construire des introductions où j'expliquais le rapport au présent, même si c'était encore ténu, non frontal. J'étais habitée par ce qui se passait en France : c'était le moment où SOS Racisme dominait la scène antiraciste et contrairement à eux, j'étais absolument opposée au différentialisme. La marche des beurs et toutes sortes d'événements

\footnotetext{
2. Les Mots de l'histoire. Essai de poétique du savoir, Paris, Seuil, 1992. Réédité par la suite sous le titre Les Noms de l'histoire.

3. «Éloge de l'anachronisme en histoire», Le Genre humain, 27, 1993 : L'Ancien et le Nouveau, p. 23-39.

4. La thèse remaniée est publiée sous le titre, L'Impossible Citoyen : l'étranger dans le discours de la Révolution française, Paris, Albin Michel, 1997.
} 
faisaient que mon travail était en prise avec le présent; c'est cela qui m'intéressait et me donnait l'énergie de fouiller les discours parlementaires et la série $\mathrm{C}$ des Archives nationales. Mais par ailleurs la Révolution est un objet historique qui porte, qui est fort et dont l'archive est forte. Du coup, on se prend au jeu; je pense que sur d'autres périodes, je n'aurais pas pu me prendre au jeu de la même manière. Ensuite, je mets en place un projet sur les émotions politiques, je rentre au CNRS en 1995 et, dès novembre, je participe au mouvement de novembredécembre 1995, à la pétition Bourdieu, aux enquêtes sur le vif. Le rapport au présent devient alors très concret!

LABYRINTHE - Quel est, dans l'écriture, votre rapport aux institutions et à leurs normes?

SOPHIE WaHNICH - Après la publication de L'Impossible Citoyen, j'ai reçu plusieurs commandes plutôt militantes. Moi-même, j'étais militante anti-raciste dans différents mouvements à ce moment-là. Je voulais tenir les deux bouts : à la fois réaliser un vrai travail d'historien et faire en sorte que ce travail d'historien dise quelque chose sur le présent, en transmettant vraiment quelque chose. J'avais l'espoir que la structure de l'ouvrage, les différentes manières de l'écrire produisent un plaisir du texte et un désir de savoir. Une somme de six cents ou neuf cents pages, cela donne au lecteur un sentiment océanique. Le savoir est alors difficile à transmettre, le lien avec le présent des lecteurs plus difficile. Momentanément : cela ne veut pas dire qu'il est inutile, car ensuite on pourra puiser dedans, il pourra être «réanimé»... Et puis on n'en décide qu'en partie, je viens d'écrire un gros livre intimidant de cinq cents pages, j'en ai été moi même surprise! Mais à ce moment-là mon objectif était de faire une histoire critique disponible. J'ai aussi bien sûr été aidée par les réflexions de Jacques Rancière, et par le travail de Régine Robin. L'un et l'autre posaient la question du récit historique comme genre littéraire, de la manière dont l'historien peut tenter de trouver une écriture pour le présent.

Écrire sous la forme de l'essai court, comme pour La Liberté ou la $M o r t^{5}$, permet en partie de s'affranchir des contraintes institutionnelles, d'une manière dont on ne mesure jamais les conséquences. Mais la matière de cet essai était parue sous une forme savante institutionnelle,

5. Paris, La Fabrique, 2003. 
en partie dans les Annales et en partie dans le Genre humain ${ }^{6}$. Les articles savants étaient parus et je savais qu'ils avaient été lus ou qu'ils seraient lus par des savants. Avec un petit essai, l'objectif était de trouver des médiations qui permettent d'atteindre un autre public. Et pour le coup, l'essai a très bien fonctionné; il a en effet atteint un autre public.

LABYRINTHE - Vous avez aussi publié dans des revues moins savantes, comme Lignes, par exemple.

Sophie WaHNich - Effectivement, j'avais écrit un article sur la Terreur paru dans Lignes ${ }^{7}$. J'aime beaucoup cette revue, à la fois littéraire et philosophique, et qui s'adresse encore à un autre public. Mais Lignes m'avait surtout permis d'expérimenter une autre écriture, avec cet article et avec un autre plus important sur les «bribes d'émotions populaires », sur les grèves de 1995, les mouvements de chômeurs, les mouvements de sans-papiers de 1997. C'était mon premier article dans Lignes ${ }^{8}$. C'était juste après la sortie de mon livre L'Impossible Citoyen, cela n'avait aucun rapport avec la notion d'étranger, mais beaucoup avec la question de la mise en scène de l'archive immédiate et sa mise en relation avec celle de l'archive révolutionnaire.

Lignes m'avait permis de sortir des revues scientifique et de l'écriture savante très normée. Il faut certes connaître cette langue commune et savoir en user dans son champ professionnel, mais immédiatement après la thèse ma priorité psychique était ailleurs. Dès que j'avais l'occasion de trouver des voies traversières, je me précipitais dessus.

LABYRINTHE - Comment sont apparus dans votre travail ces objets différents, non textuels, que sont par exemple les musées ou le cinéma? Est-ce un hasard?

Sophie WAHNich - Sans doute pas. Mais il y a une part de hasard dans la connexion. Quand je suis rentrée au CNRS, j'ai été nommée à Amiens dans la Somme. Un jour, en me promenant, je suis allée au musée de Péronne. Et ce musée m'a agacée. Sans savoir bien nommer

\footnotetext{
6. «La terreur comme fondation, de l'économie émotive de la terreur», Annales, histoire sciences sociales, $57, \mathrm{n}^{\circ} 4$, juillet 2002, p. 889-914; et «Recevoir et traduire la voix du peuple», Le Genre humain, 40-41, 2003 : Qui veut prendre la parole?, Marcel Detienne (dir.), p. 349-372.

7. «Terreur révolutionnaire et terrorisme. Rémanences rétiniennes et troubles de la vision», Lignes, nouvelle série, $\mathrm{n}^{\circ} 8$, mai 2002, p. 147-167.

8. «Bribes d'émotions (populaires)», Lignes, n³7, mai 1999, p. 160-184.
} 
les raisons de cet agacement, je me disais que quelque chose n'allait pas. Peu après, il y a eu un appel d'offre sur «l'identité européenne en question» et ma collègue Mireille Gueissaz m'a invitée à proposer quelque chose. J'ai alors pensé à ce musée, qui est présenté comme un musée européen de l'histoire de la guerre de 1914. Dans ce lieu, des historiens avaient tenté de fabriquer de l'identité européenne, avec beaucoup d'énergie et d'une manière clairement affirmée. Pour moi, c'était un musée de l'identité européenne vue par les Français. Se sont alors joints à nous Pieter Lagrou et Isabelle Benoît ${ }^{9}$. À ce moment-là, Annette Becker nous a mis en contact avec d'autres interlocuteurs d'une manière très ouverte et sans être d'accord avec notre point de vue. Par contre, je n'ai jamais eu de contact avec Stéphane AudouinRouzeau. Je retrouvais dans ces musées la question du nazisme, des fascismes, d'une manière détournée et donc possible, parce que c'était les représentations aujourd'hui et non un travail direct sur l'extermination en tant que telle.

C'était aussi une manière de sortir de l'analyse de discours stricte; j'avais eu quelques critiques au moment de la soutenance, en particulier de Paolo Viola qui me rappelait à la factualité et à la dimension anthropologique. Mon projet CNRS portait sur les émotions, ce qui était déjà une manière de se décaler du côté du lien, ou de l'absence de lien, qu'on pouvait faire entre histoire, langage, anthropologie. Jacques Guilhaumou était absolument mécontent : en fait, il voyait bien les enjeux, et il voyait bien que c'était une manière de rencontrer les limites de l'analyse de discours. L'histoire des émotions ne peut pas s'enfermer dans l'analyse de discours, même si la plupart des documents restent des discours. Mais il y a aussi d'autres documents possibles, comme les images ou les représentations au musée - dont on peut faire une analyse discursive, sauf que si on se contente du discursif on manque le musée. Donc on est obligé de passer par d'autres moyens, qui sont du bricolage. De ce point de vue, plus que les sociologues ou les philosophes, ce sont les anthropologues qui sont les plus proches des historiens. Ils sont aussi dans l'empirique, dans le bricolage et en même temps dans la thick description. C'est-à-dire

9. Cette recherche a notamment débouché sur une publication collective : Mireille Gueissaz, Sophie Wahnich (dir.), Les Musées d'histoire des guerres du XXe siècle, des lieux du politique ?, Paris, Kimé, 2000. Puis Fictions d'Europe. La guerre au musée, en Allemagne, en France et en Grande-Bretagne, Paris, éditions des Archives contemporaines, 2002. 
qu'ils sont aussi habités par des enjeux théoriques : il ne s'agit pas d'une description plate, où on ne sait pas bien où l'on va et où on décrit tout, comme Borges quand il parle de faire des cartes à l'échelle 1/1.

La matière muséale offrait aussi une manière d'être dans le présent illico presto, c'est-à-dire dans le présent des institutions de politique publique, de leur financement, des publics, du travail avec des élèves qui viennent visiter. On avait organisé un travail avec une enseignante d'histoire de Saint-Nazaire qui avait emmené ses classes. On avait suivi ses classes, on avait interrogé les élèves, on avait enfin travaillé avec du vivant. La traversée passé-présent, c'est aussi quelque chose d'assez douloureux, car c'est aller visiter les morts, et aller visiter les morts... Il $\mathrm{y}$ a trop de morts... Ils pèsent et ils ne pèsent pas. Lesquels visiter et comment les visiter? Je n'ai pas la jouissance de Michelet à aller voir les morts. J'ai eu besoin de quelque chose de très ancré dans le présent pour pouvoir poursuivre un travail finalement douloureux, un exercice difficile malgré cette vivacité de l'archive révolutionnaire.

LABYRINTHE - Dans la question des émotions telle que vous l'abordez, il y a toute une dimension esthétique. Pour ces questions-là, est-ce aussi chez les anthropologues que vous avez trouvé des compagnons de pensée, ou plutôt chez les philosophes?

Sophie WaHNich - Ce n'est pas directement chez les anthropologues, il faut être honnête. Mais par contre, c'était très accueillant d'être chez les anthropologues, parce que c'est une zone d'abri. Ils sont suffisamment empiristes pour comprendre qu' on le soit en tant qu'historiens ; c'est donc beaucoup moins difficile d'être avec des anthropologues que d'être avec des sociologues. Pour les historiens, je me souviens qu'à la fin de ma thèse, je passais pour assez théoriciste, mais pour les sociologues j'étais d'un empirisme détestable. Les anthropologues ne me demandaient pas si j'étais ceci ou cela, en tout cas pas au $\operatorname{LAIOS}^{10}$ où je suis depuis 1999, parce que c'est un laboratoire très ouvert du point de vue des enjeux théoriques : les gens sont dans une quête de compréhension et font usage d'outils, ils n'ont pas du tout de fétichisme des outils théoriques en soi.

C'est aussi mon rapport à la théorie : ne pas oublier que ce sont des modélisations et que ces modélisations soit étayent soit font obstacle.

10. Laboratoire d'anthropologie des institutions et des organisations sociales. 
Quand elles étayent c'est parfait, quand elles font obstacle, il faut réussir à s'en débarrasser. Chez Marc Abélès par exemple, si on ne veut pas voir les enjeux théoriques dans son écriture, on ne les voit pas. Pourtant, dans tous ses ouvrages, les enjeux théoriques sont là, en particulier dans celui sur les nouveaux riches dans la Silicon Valley qui est une reprise des enjeux théoriques du don ${ }^{11}$. Le LAIOS était un lieu institutionnel et théorique qui me correspondait bien. Je conjugue tout cela au passé, parce que j'ai l'impression qu'il y a une étape nouvelle, qui n'est pas liée aux chercheurs du LAIOS en tant que tels mais à la configuration en France de la recherche. Maintenant la conjoncture est extrêmement individualiste. Comme on fonctionne sur appels d'offre avec l'ANR, il y a des «porteurs de projet» et le «porteur de projet» compte pour $20 \%$ de l'évaluation du projet.

Si j'avais travaillé sur les musées dans ce contexte-là, je n'aurais peut-être obtenu aucun soutien, parce que je n'étais pas connue du tout. J'ai eu la subvention sur la teneur du projet, pas sur mon nom, surtout que je n'étais pas spécialiste des musées. Cette spécialité n'existait d'ailleurs pas à l'époque et les historiens s'opposaient à sa création, ils disaient qu'on n'allait pas donner de l'argent à des gens pour qu'ils aillent se balader dans les musées. À l'époque, le projet avait donc été soutenu par les anthropologues. Dans le cadre des projets ANR, on assiste à un renouvellement de l'inféodation : les porteurs de projet agrègent des chercheurs professionnels, des postdoctorants, des doctorants; ce sont de grosses machines institutionnelles et les enjeux de recherche en tant que tels deviennent, non pas mineurs mais moins innovants. Aujourd'hui, il est presque impossible de concevoir une valorisation des liens entre chercheurs si on ne les met pas sur un plan institutionnel. Tout ceci alourdit énormément les procédures et rend les laboratoires plus fictionnels.

Mais pour en revenir aux catégories d'esthétique, je les ai puisées plutôt chez les philosophes, comme Jacques Rancière à nouveau; je pouvais aussi en discuter avec mon collègue Henri-Pierre Jeudy qui se pose souvent des questions analogues et très vives sur l'esthétique.

11. Marc Abélès, Les Nouveaux Riches. Un ethnologue dans la Silicon Valley, Paris, Odile Jacob, 2002 . 
LABYRINTHE - Quelle peut être l'influence de l'intervention historienne sur l'effectivité $d u$ "partage du sensible»?

SOPHIE WAHNICH - Je n'ai pas beaucoup d'illusions là-dessus! Il y avait un entretien de Gérard Noiriel dans Le Monde où il disait : «il faut reconnaître qu'on ne sert à rien, qu'on en est très triste mais c'est comme ça». Il dit que si on veut transmettre sa préoccupation actuelle, il faut passer par le spectacle vivant ou par le cinéma. Je pense effectivement que les textes des historiens aujourd'hui, quels qu'ils soient, pour des raisons de configuration de la place du livre dans la société, ne transmettent plus que difficilement. On est dans une crise de la conscience historique. Quand l'histoire est «à la mode», qu'il y a une forte conscience historique, les livres d'histoire se vendent bien, se lisent. Aujourd'hui nous sommes dans une configuration inverse, dans un post-modernisme complet, où on peut citer n'importe quoi dans n'importe quel ordre. Il faut donc trouver d'autres moyens pour transmettre ce qui a à voir avec cette conscience historique. De ce point de vue, mes prochains travaux se feront avec le cinéma, avec l'opéra ${ }^{12}$. Ils ne seront pas uniquement du côté du livre d'histoire, même si je suis ravie d'avoir trouvé un bon éditeur pour mon prochain livre. Je pense que si on se contente du livre d'histoire, il ne faudra pas se lamenter : on sait que ça ne sert pas directement.

Le monde politique est anti-intellectuel, y compris à l'extrêmegauche. Aujourd'hui, on supporte les intellectuels, mais on ne les prend pas au sérieux. Y compris chez les intellectuels eux-mêmes. Par exemple, j'aime bien les livres de Daniel Bensaïd, j'aime bien son écriture, j'y trouve des enjeux intéressants, des références qui me sont utiles. Mais si je discute avec lui, philosophe et militant de toujours, dirigeant de la LCR, j'observe que, pour lui, le travail intellectuel n'est pas un enjeu dans le mouvement social et politique. Je ne sais pas s'il a tort ou raison! Mais c'est un symptôme d'anti-intellectualisme. Autrement dit, la pensée de Rancière, par exemple, n'est pas du tout une pensée incorporée dans la manière de faire de la politique aujourd'hui. Le poids des mots plutôt que le poids des salaires, ça ne fait pas partie du discours syndical par exemple. D'où le sentiment qu'on peut avoir, de la vanité de notre travail. Aujourd'hui, il n'y a pas

12. Sophie Wahnich a notamment participé comme scénariste à l'expérience filmée de Dominique Cabrera intitulé Le Beau Dimanche, 2007. 
d'espace de réception construit pour lui. Il doit donc passer par d'autres moyens, comme le cinéma, les musées, les spectacles vivants.

LABYRINTHE - Ce serait donc une politique qui ne passerait plus par les mots, mais plutôt par l'esthétique et l'émotion?

Sophie Wahnich - Non, ce sont les mots aussi. Si on fait un film, par exemple, il y a évidemment la question de la scénarisation. On est tout de même dans une matière langagière d'archive. Et à partir de cette matière langagière d'archive, on se demande comment on va pouvoir faire une matière langagière de scénario. On retrouve, dans la matière de la langue, le passage passé/présent. En tant que pratique théorique, pour moi, ça ne change rien, c'est le même enjeu. En termes de pratique intellectuelle, c'est enthousiasmant car c'est se mettre enfin dans la pâte de la langue, non pas la regarder pour la décrire mais la prendre véritablement en main. Donc pour moi, ce n'est pas les émotions contre les mots, c'est savoir faire circuler des émotions dans les mots pour que les mots redeviennent audibles. C'est en cela que je ne crois pas à une science qui récuserait toute dimension sensible dans son écriture. Là-dessus je suis assez d'accord avec Arlette Farge.

Pendant la campagne présidentielle, il y a eu des discours alarmistes qui pointaient le jeu de Ségolène Royal ou de Nicolas Sarkozy dans le registre émotionnel. Je pense que ce qui est alarmant, ce n'est pas de jouer sur le registre émotionnel, c'est de ne pas articuler l'argumentatif et l'émotionnel. Dans une certaine mesure, quand on a un discours argumentatif «sans émotion» (ce qui était plus ou moins le discours de Jospin - sur le mode «je suis très sérieux», «je ne ferai pas d'effet» et «je ne gouvernerai pas avec la rue»), surgit aussi un discours inverse, comme celui de la démocratie participative. Sans y être explicitement favorable dans ses formes actuelles, il faut reconnaître qu'il y a là quelque chose d'audible concernant la nécessité de retrouver le lien émotionnel. Et c'est en retrouvant ce lien émotionnel que des arguments pourront à nouveau passer. Je pense qu'il n'y a rien là d'alarmant. Il s'agit simplement de dire que les limites d'une raison qui a voulu, pour des raisons historiques, se détacher des émotions, sont atteintes. Il faut réintroduire du sensible et c'est une des manières possibles de le faire. Je suis optimiste; cela ne m'arrive pas souvent, mais là, j'ai envie de l'être. 
Ce qui est négatif, c'est qu'il peut y avoir à nouveau un usage démagogique des émotions, du fait d'un refus de les penser. Dans le travail que j'ai entrepris, j'ai essayé de penser des émotions dans le politique qui ne seraient pas les cathédrales de lumières du nazisme, qui ne sont pas l'esthétisation du politique décrite par Benjamin ou l'aliénation telle que l'a décrite Freud dans les phénomènes d'identification. Des émotions qui seraient un étayage pour pouvoir être au monde politique, comme l'a décrit Jacques Rancière. Néanmoins, Rancière décrit surtout les apologues, pas la matière de l'événement. J'essaie quant à moi de travailler la matière de l'événement révolutionnaire. J'essaie de saisir ce qui se joue de symbolique, comment on bricole des choses pour retenir la violence, comment on invente des formes pour être dans la vie plutôt que dans le mortifère, et comment on essaye de contrôler tous les dangers liés aux émotions. Ce qui est inquiétant aujourd'hui, ce n'est pas l'usage des émotions mais le fait qu'il n’y a pas de pensée des émotions.

LABYRINTHE - Quelle écriture historienne inventer pour répondre à ce défi? Votre récent texte "Au pays des trains fantômes» est-il le modèle de ce que vous cherchez à faire en ce moment ${ }^{13}$ ?

Sophie WahNich - Cet article est une tentative de répondre à la question : qu'est-ce que serait une poétique du savoir du rapport passé/ présent, pour le présent? Si on se contente de l'écriture savante, hypercodifiée, en particulier par les revues - mais certains rêvent d'une codification plus dure encore avec une langue normale comme aux Etats-Unis - l'article devient une construction rhétorique figée où on va pouvoir citer des références académiques. C'est la victoire du splendide isolement des champs à la manière de Pierre Bourdieu. L'écriture des dissertations et des concours m'a intéressée car elle est formatrice. Mais quand on a fait ça un temps, on se dit qu'il faut peut-être passer à autre chose, chercher des manières de faire qui rendent plus poreux les champs savants, artistiques, politiques. Depuis l'article dans la revue Lignes dont j'ai parlé tout à l'heure, je suis à la recherche d'une autre écriture, par fragment, par concaténation et choc de différentes choses hétérogènes. Pour essayer d'être honnête avec la manière dont

13. «La Révolution française au pays des trains fantômes», Contretemps, 11, 2004, p. 140-150, également disponible sur le site http://revolution-francaise.net/ [consulté le 6 décembre 2007]. 
les connexions se font dans la pensée. Par exemple, lorsqu'on travaille dans l'archive, on se dit que la question du droit d'ingérence, telle qu'elle s'est posée en ex-Yougoslavie, était déjà réfléchie par les révolutionnaires quand ils disaient qu'ils ne fallaient pas se mêler des révolutions des autres ${ }^{14}$.

Néanmoins, il ne faut pas confondre la concaténation stylistique et la concaténation du passé et du présent dans une écriture tout à fait conventionnelle.

En écrivant on fait des tentatives, des expériences. Ce n'est pas immédiatement recevable, mais au moins on œuvre.

LABYRINTHE - Sur le traitement des émotions, à vous lire, on hésite entre deux orientations : une compréhension du rôle des émotions comme "effectuation» de la question de la souveraineté du peuple, d'une part; et en même temps, d'autre part, vous injectez la question du contrôle des émotions.

Sophie Wahnich - Dans les émotions, il y a l'articulation entre du pulsionnel, de l'esthétique et du discours. Et le pulsionnel est toujours un peu dangereux. Une des choses qui m'a le plus intéressée dans le travail sur 1792, c'est de rencontrer une conscience de ce danger, du danger de l'emballement de la violence. Dans la logique populaire, quand il y a une altercation, il faut qu'il y ait des amis pour celui qui dit «retenez-moi ou je fais un malheur». Je pense que cette conscience du «retenez-moi ou je vais faire un malheur» est à la fois réflexive du danger tout en offrant la possibilité de l'exprimer. Cela peut être très expressionniste : dans une pièce de Brecht, il y aurait un gars en colère avec d'autres gars qui le retiennent. Il faut trouver la ligne de crête qui permet à la fois d'énoncer cette souveraineté populaire sans qu'elle puisse basculer dans le malheur, à savoir la violence sans retenue. Les institutions, au sens civil, de l'amitié, de l'hospitalité, ou au sens plus classique des appareils sociaux et politiques, magistrats, directoires, municipalité, ont cette fonction.

14. Voir Sophie Wahnich, «La Yougoslavie hante l'Europe. À propos de quelques manifestations artistiques du spectre», dans Gabrielle Varro (dir.), Regards croisés sur l'Ex-Yougoslavie : des chercheurs face à leurs objets de recherche et aux événements sociaux et politiques, Paris/Budapest/ Turin, L'Harmattan, 2005, p. 55-82. 
LABYRINTHE - Mais est-ce que ce contrôle ne réinjecte pas une contradiction en face du principe de souveraineté?

Sophie Wahnich - Cela dépend si on pense que la souveraineté populaire est directe, ou si on pense que c'est une souveraineté qui n'est ni anti-intellectualiste, ni anti-parlementaire. Dans la période révolutionnaire, elle n'est pas anti-intellectualiste. On a une conscience très claire que des intellectuels comme Robespierre, Saint-Just, etc., défendent le mouvement populaire. Il n'y a pas de contradiction entre être dans la souveraineté populaire et choisir des porte-parole, ce qui n'est plus le cas aujourd'hui. Aujourd'hui, il n'y a pas de porte-parole qui ne soit pas dans la confiscation. Même si Robespierre a pu être aussi dans cette confiscation, à un moment donné, il est autorisé à être le porte-parole du peuple parisien ou du peuple français. On est dans une tension entre ce qu'on a à dire et la possibilité que le porte-parole le traduise. Cette question de la traduction est fondatrice de cette retenue. Quand il n'y a plus de traduction, que le peuple est livré à luimême et qu'il n'y a plus d'étape de construction de l'espace public où on puisse à la fois être dans le discours, les émotions, l'argumentation, pour produire une loi juste, alors - c'est ce que j'ai essayé de montrer - on est dans la violence nue de la souveraineté. Aucun des révolutionnaires en 1789, 1791 ou en 1792, ne le souhaite. Les massacres de septembre sont des malheurs. Madame Julien de la Drôme écrit alors : «je jette ici, d'une main tremblante, un voile sur les crimes qu'on a forcé le peuple à commettre par tous ceux dont il est depuis trois ans la triste victime». C'est terrible d'avoir poussé le peuple à devoir se faire justice lui-même. De la même manière, on pourrait plaindre les résistants d'avoir dû affronter le «faire-mourir». Dans L'Armée des ombres $^{15}$, quand il faut faire mourir le traitre, il y a un personnage qui pleure, les autres sont dans un état terrible.

LABYRINTHE - Est-ce que le statut du pulsionnel est différent aujourd'hui, avec l'intériorisation de la norme et de la discipline?

SophiE WaHNich - Je ne suis pas sûr que le pulsionnel soit tellement institutionnalisé. Il y a quelque chose de transhistorique dans le pulsionnel. Une accumulation discontinue de pulsionnels individuels produit après coup la désolation de la foule ivre de colère. Ces appré-

15. Film de Jean-Pierre Melville (1969). 
ciations que l'on trouve dans Taine ne sont pas fausses. Du côté gauche, on va plaindre la foule d'avoir été mise dans cet état-là, comme on peut plaindre une femme d'avoir un mari trop lâche et qui la pousse à devenir hystérique - je pense ici au film de René Allio, Moi, Pierre Rivière. Du côté droit on va accuser les émeutiers de ne pas avoir d'autre langage. Je pense qu'on peut déplorer dans les deux cas qu'il n'y ait pas d'autre langage. Mais on peut aussi considérer qu'il y a une configuration historique qui a produit cette situation et cette factualité. Le renoncement à transmettre d'autres langages, à politiser, à produire des institutions est à analyser en amont des phénomènes émeutiers.

Que signifie le feu des émeutes ? Cela a quelque chose à voir avec l'Enfer, avec le jusqu'auboutisme. Quand on brûle, on donne une symbolique de l'anéantissement, de la terre brûlée. Il y a ainsi toute une série de métaphores littéraires qui permettent de penser la situation des émeutes (notamment celles de 2005), qui n'ont pas été utilisées. Pousser les gens à la terre brûlée, c'est ne pas leur avoir donné d'autres outils. On peut donc les plaindre, comme Victor Hugo plaint les communards d'avoir brûlé une bibliothèque ${ }^{16}$. Mais pourquoi a-t-on des gens qui n'ont pas conscience que brûler une bibliothèque, c'est terrible aussi pour eux? Personne ne peut se féliciter qu'on brûle une bibliothèque ou une école. Mais les manières d'évaluer les responsabilités diffèrent : le côté gauche déplore une situation historique, et le côté droit accuse les émeutiers.

16. Voir Victor Hugo, «À qui la faute? », dans L'Année terrible. Euvres complètes, t. XII, Paris, Hetzel, 1880, p. 307-330 (poème écrit en exil après l'incendie des Tuileries et de sa bibliothèque par les Communards). 